\title{
Numerical investigation of swirl flow inside a supersonic nozzle
}

\author{
E. Eslamian, H. Shirvani \& A. Shirvani \\ Faculty of Science and Technology, Anglia Ruskin University, UK
}

\begin{abstract}
This study reports the Computational Fluid Dynamics (CFD) results of a swirling flow induced by introducing a helical insert inside a supersonic nozzle. The CFD simulation shows a very complex unsteady, non-axisymmetric flow pattern for the swirl flow inside the nozzle. The flow is investigated by solving the Reynolds averaged Navier Stokes (RANS) equations with k- $\omega$ and Reynolds Stress Model (RSM) turbulence models to predict the flow patterns and the type of swirling flow. Computations are conducted for a range of nozzle pressure ratios with and without swirl inside the converging-diverging nozzle. The study has revealed a new understanding and data for flow features such as shock location, mass flow rate and anisotropic turbulence.
\end{abstract}

Keywords: swirl flow, supersonic nozzle, flow separation, CFD.

\section{Introduction}

Investigation of supersonic flow inside converging-diverging (C-D) nozzles has been the subject of several numerical and experimental studies in the past $[1,2]$ but there is not that much research on the effect of swirl flow inside C-D nozzles. Swirling flows which are very common in technical applications, such as turbo machinery, cyclones or separators, and they require sophisticated modeling.

The effect of swirl inside a nozzle can improve the mixing features of the flow by increasing turbulence and vorticity in the nozzle, which can be useful in combustion injectors and sand blast techniques; also swirl flow will change the shock structure and its interaction with the boundary layer and create a larger separation zone at the exit of a nozzle. These viscous and compressible phenomena affect the flow behavior inside and outside of a nozzle. Since 
experimental data for the swirl flow inside a high speed nozzle are scare, numerical results are vital for understanding the flow and for further analysis.

In this study the finite volume method is utilized (Fluent), to solve the governing equations of flow.

\section{Numerical analysis}

\subsection{Nozzle geometry}

The dimension of the nozzle with the flow inside which has been investigated is shown in Figure 1a. The length of the nozzle is $200 \mathrm{~mm}$ and it has three sections. The first $64 \mathrm{~mm}$ is the convergence section, then comes a $16 \mathrm{~mm}$ section with constant $11 \mathrm{~mm}$ diameter and this is followed by the divergence section with length $120 \mathrm{~mm}$ and outlet diameter of $15 \mathrm{~mm}$. The computational domain which consists of the helical inlet and the nozzle is illustrated in Figure 1b. The helical section, Figure 1c, is used to create swirl flow inside the nozzle. The helical section has a $31.75 \mathrm{~mm}$ diameter with length of $76.45 \mathrm{~mm}$; the spiral part has two revolutions with a start angle of 45 degrees.

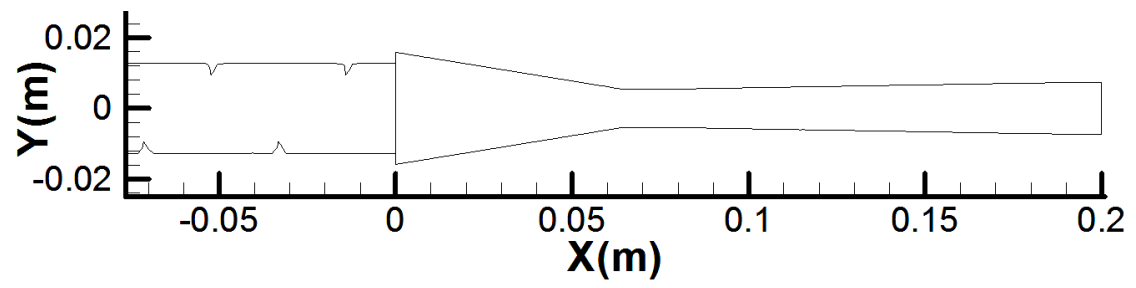

(a)
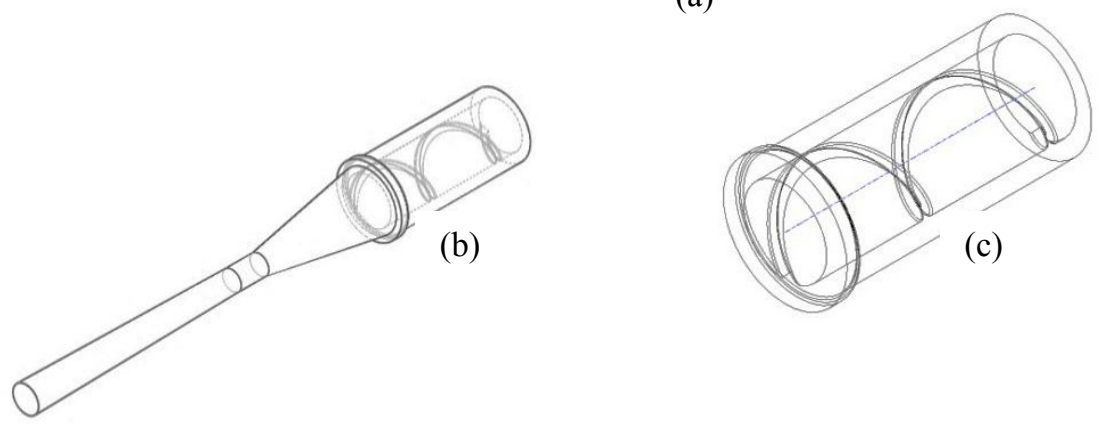

Figure 1: Geometry and dimensions of the computational domain.

\subsection{Numerical model}

Turbulence modelling is still one of the main problems of Computational Fluid Dynamics (CFD). The models mostly used for fluid phenomena are the standard k- $\varepsilon$ and k- $\omega$ models. These Reynolds averaged Navier Stokes (RANS) models give adequate results for isotropic turbulence flows, and have been validated 
with many experimental tests (Wilcox [3]). However, as we have highly swirling flow inside nozzle, there will be a considerable degree of anisotropy in the stress and dissipation tensors which causes a highly anisotropic eddy viscosity (Yajnik and Subbaiah [4]), so the isotropic turbulence model will not give precise results. To be able to capture anisotropic turbulence inside a nozzle we have to consider all Reynolds stress equations. The highest accuracy in turbulence is acheived by Direct Numerical Simulations (DNS), but this needs huge processing power. Large Eddy Simulations (LES) is another major turbulence model which lately has begun to grow in popularity and many academic and industry users deploy it for their research and applications. LES resolves all anisotropic turbulent structures both in time and space, and just leaves the small scales to be modelled by simple turbulence models (Ogor et al. [5]). However, we still need a lot of precessing power and a very fine mesh to use LES modelling.

The RANS models for anisotropic turbulence are limited to the modified k- $\varepsilon$ and Reynolds Stress Model (RSM). We have used a realizable k- $\varepsilon$ model, which contains an alternative formulation for eddy viscosity and uses a modified transport equation for dissipation rate (Shih et al. [6]) but the RSM model solves all six transport equations. In this research, the RSM gave more consistent results, and for a realizable k- $\varepsilon$ method we need to carry out more research with a higher order scheme; therefore the focus is more on the RSM and the results shown here are based on the RSM.

For the RSM a second oreder upwind scheme is applied to the flow equations, but the turbulent kinetic energy, turbulent dissipation rate and Reynolds Stress are considered first order upwind.

The initial simulations were conducted with a steady method, but the convergence history of residuals showed unsteadiness which is in agree with the results of Xiao et al. [7]; the main reason for this is the separation of the flow at the exit of the nozzle. Therefore a transient solver is considered for the flow with 1e-5 time step intervals.

The air in the nozzle will reach $M a>1$, so compressibility is one of the main factors for the flow solver. The density based solver in Fluent gives more accurate results and has better convergence rate for compressible flows. It also captures more precicely the shock waves in the nozzle .

\subsection{Governing equations}

The density based solver from Fluent has been used to solve the governing equations of the flow. The conservative integral form of the continuity, momentum and energy equations for a single component fluid in an infinitesimal control volume is (Fluent [8]):

$$
\frac{\partial}{\partial t} \int W d V+\oint[F-G] \cdot d A=\int H d V
$$

where the vectors $W, F$ and $G$ are defined as: 


$$
W=\left\{\begin{array}{c}
\rho \\
\rho u \\
\rho v \\
\rho w \\
\rho E
\end{array}\right\}, F=\left\{\begin{array}{c}
\rho v \\
\rho v u+P \hat{\imath} \\
\rho v v+P \hat{\jmath} \\
\rho v w+P \hat{k} \\
\rho v E+P v
\end{array}\right\}, G=\left\{\begin{array}{c}
0 \\
\tau_{x i} \\
\tau_{y i} \\
\tau_{z i} \\
\tau_{i j} v_{j}+q
\end{array}\right\}
$$

The vector $H$ contains all of the source terms such as the body forces. In the above equation $\rho$ is density, $v$ is velocity, $E$ is total energy per unit mass and $P$ is pressure. $\tau$ and $q$ are the viscous stress tensor and heat flux, respectively. In the density based solver of Ansys Fluent, to overcome the poor convergence rate of high speed flows, a preconditioning technique has been deployed. The preconditioning technique modifies the time derivative term of equation (1) by multiplying it with a preconditioning matrix. This will rescale the acoustic speed of the governing equations in order to mitigate the numerical stiffness encountered in low Mach numbers.

\subsubsection{Turbulence equations}

In Reynolds averaging the variables of exact N-S equations are substituted with mean and fluctuation components. By substituting $u_{i}=\bar{u}_{l}+u_{i}^{\prime}$ into the momentum equation, where $\bar{u}_{\imath}$ is the mean and $u_{i}^{\prime}$ is the fluctuating velocity components, the time average of the momentum equation can be shown as:

$\frac{\partial}{\partial t}\left(\rho u_{i}\right)+\frac{\partial}{\partial x_{j}}\left(\rho u_{i} u_{j}\right)=-\frac{\partial P}{\partial x_{i}}+\frac{\partial}{\partial x_{j}}\left[\mu\left(\frac{\partial u_{i}}{\partial x_{j}}+\frac{\partial u_{j}}{\partial x_{i}}-\frac{2}{3} \delta_{i j} \frac{\partial u_{l}}{\partial x_{l}}\right)\right]+\frac{\partial}{\partial x_{j}}\left(-\rho \overline{u^{\prime}{ }_{l} u_{j}^{\prime}}\right)$

In order to close equation (3), the Reynolds stress term $\left(-\rho \overline{u^{\prime}{ }_{\imath} u_{j}^{\prime}}\right)$ must be modelled. In this study we have used the RSM, which uses the exact transport equation for the transport of the Reynolds stresses, $-\rho \overline{u^{\prime}{ }_{l} u_{j}^{\prime}}$, which can be written as:

$$
\frac{\partial}{\partial t}\left(\rho \overline{u^{\prime} u_{j}^{\prime}}\right)+C_{i j}=D_{T, i j}+D_{L, i j}+P_{i j}+G_{i j}+\phi_{i j}-\epsilon_{i j}+F_{i j}
$$

where $C_{i j}$ is the convection term, $D_{T, i j}$ stands for turbulent diffusion, $D_{L, i j}$ is the molecular diffusion term, $P_{i j}$ equals the stress production, $G_{i j}$ is buoyancy production, $\phi_{i j}$ stands for pressure strain, $\epsilon_{i j}$ is the dissipation and $F_{i j}$ is production by the system rotation. Four parameters, $D_{T, i j}, G_{i j}, \phi_{i j}$ and $\epsilon_{i j}$, have to be modelled in order to close equation (4), but the rest of the parameters do not required modelling. The turbulent diffusion $\left(D_{T, i j}\right)$ has been modelled with the simplified model of Daly and Harlow [9]:

$$
D_{T, i j}=\frac{\partial}{\partial x_{k}}\left(\frac{\mu_{t}}{\sigma_{k}} \frac{\partial \overline{u_{l}{ }_{l}{ }^{\prime \prime} \jmath}}{\partial x_{k}}\right)
$$

where $\mu_{t}$ is the turbulent viscosity. The pressure strain term has been modelled according to the proposal by Gibson and Launder [10]. Buoyancy production $\left(G_{i j}\right)$ for an ideal gas is modelled as: 


$$
G_{i j}=-\frac{\mu_{t}}{\rho P r_{t}}\left(g_{i} \frac{\partial \rho}{\partial x_{j}}+g_{j} \frac{\partial \rho}{\partial x_{i}}\right)
$$

The dissipation rate $\left(\epsilon_{i j}\right)$ is modelled as follows:

$$
\epsilon_{i j}=\frac{2}{3} \delta_{i j}\left(\rho \varepsilon+Y_{M}\right), Y_{M}=2 \rho \varepsilon M_{t}^{2}
$$

In equation (7) $Y_{M}$ is an additional dilatation dissipation term according to the model by Sarkar and Balakrishnan [11].

\subsection{Numerical results}

The results for two pressure ratios $\left(P_{\text {in }} / P_{\text {out }}\right)$ of 2 and 3 are shown here. At both pressure ratios there is an overexpanded supersonic nozzle, where the shock waves occur inside the nozzle. The Mach number contours for a pressure ratio of 2 in two time steps are illustrated in Figure 2. Experimental and numerical simulations represent two separation patterns for an overexpanded nozzle, the Free Shock Separation (FSS) and Restricted Shock Separation (RSS) (Hadjadj and Onofri [12]). In FSS the separation region extends from the separation point to the end of the nozzle, but in RSS the separation zone reattaches to the surface and creates recirculation bubbles. For a pressure ratio of 2 the FSS pattern can be seen at the upper side of the nozzle wall (Figure 2).

(a)

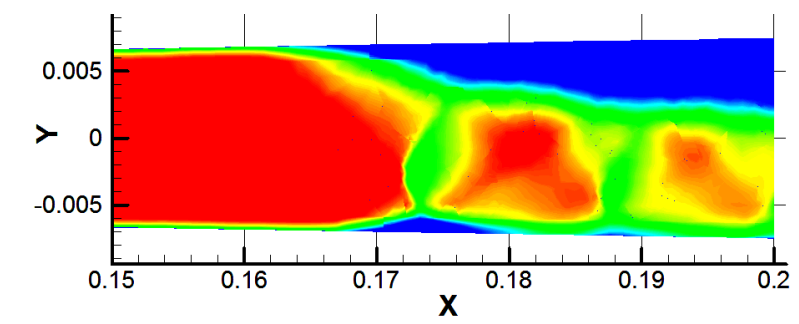

(b)
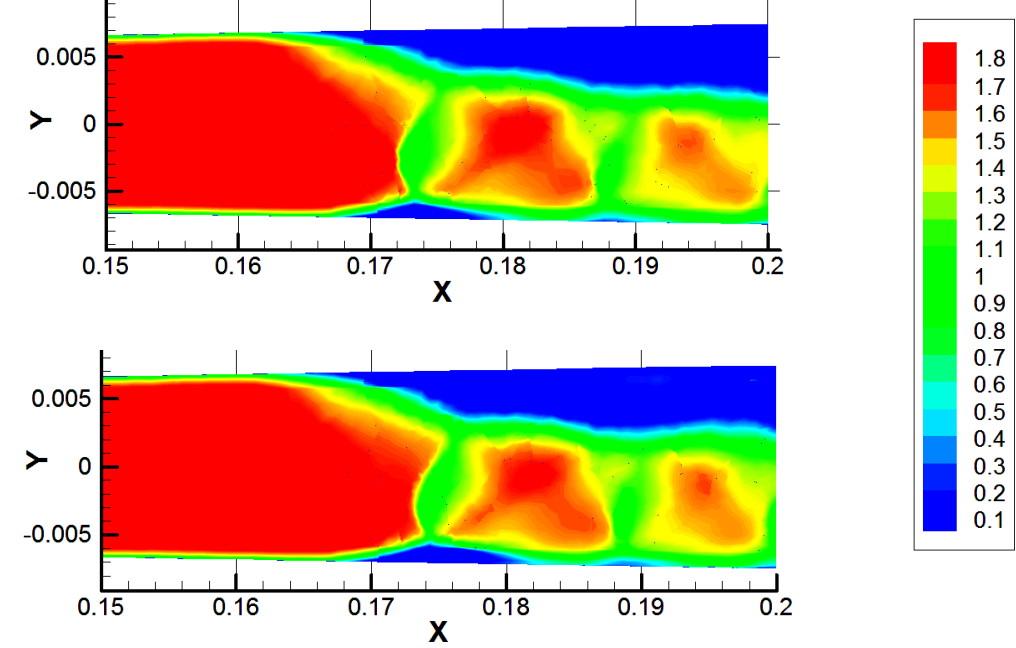

Figure 2: $\quad$ Mach number contours for a pressure ratio of 2 at (a) $t=0.8 e-2 s$ and (b) $\mathrm{t}=1.8 \mathrm{e}-2 \mathrm{~s}$.

Because of asymmetry which is due to the Coanda effect, flow tends to attach to the surface and creates a RSS pattern on the down side of the nozzle wall. The separation zone starts at the interaction point between the oblique shock wave and the nozzle wall which creates an adverse pressure gradient. Reflection of the 
oblique shock wave on the shear layer generates expansion fans. To provide more detail of the separation nozzle, Figure 3 illustrates the flow and shock wave pattern for the pressure ratio of 2. Since the separation zone is large the flow remains attached to the down side of the nozzle wall, and therefore the difference between two time steps is mostly about the change in the shear layer position.

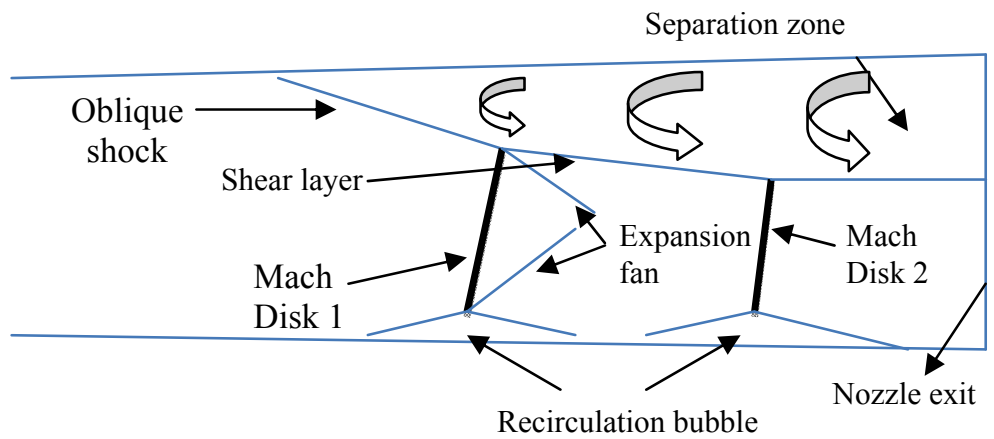

Figure 3: Schematic of the shock wave structure for the pressure ratio of 2 at the exit of the nozzle.

By increasing the pressure ratio to 3 the oblique shock waves become weaker so the pressure gradient behind it will significantly reduce and the separation point moves downstream of the nozzle. Contours of Mach number for two time steps at the exit of the nozzle are shown in Figure 4.

(a)

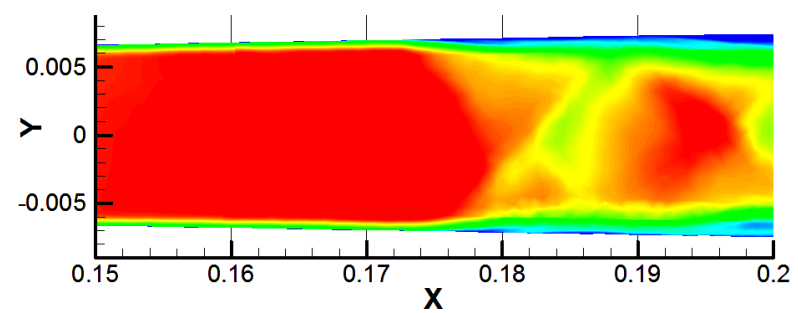

(b)
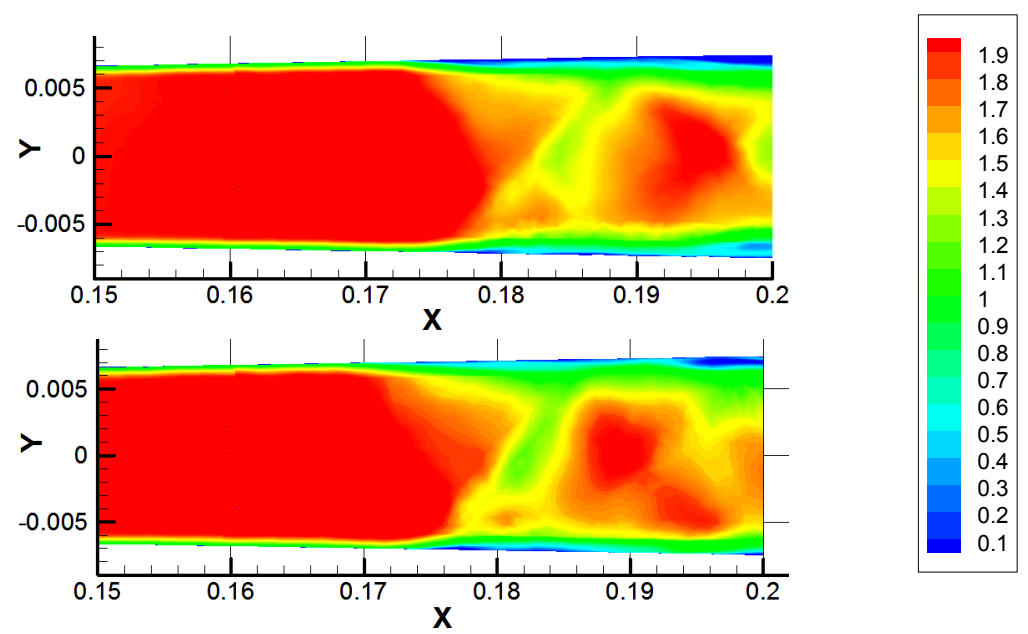

Figure 4: Mach number contours for the pressure ratio of 3 at (a) $\mathrm{t}=\mathrm{o} .8 \mathrm{e}-2 \mathrm{~s}$, (b) $\mathrm{t}=2.8 \mathrm{e}-2 \mathrm{~s}$. 
The flow pattern still remains asymmetric, and the second Mach disk moves out of the nozzle. At $\mathrm{t}=0.8 \mathrm{e}-2$, Figure $4 \mathrm{a}$, the second Mach disk is at the exit of the nozzle but in $\mathrm{t}=2.8 \mathrm{e}-2$, Figure $4 \mathrm{~b}$, it is completely out of the nozzle. Calculation of 1000 more time steps does not show any major change to the shock wave structure apart from the shear layer movement. The reflection of the shock wave on the shear layer creates expansion fans.

By adding swirl to the flow the shock wave structure becomes weaker, and instead of one strong shock wave there will be series of shock waves and expansion fans at the exit of the nozzle. This can be seen from the static pressure diagram at the centre of the nozzle which for a pressure ratio of 2 is shown in Figure 5. Also the start point of the shock wave has been moved upstream for the swirl flow. The results are compared with experimental data from Abbasalizadeh [13] for the same nozzle without the swirl that shows good agreement with CFD calculations.

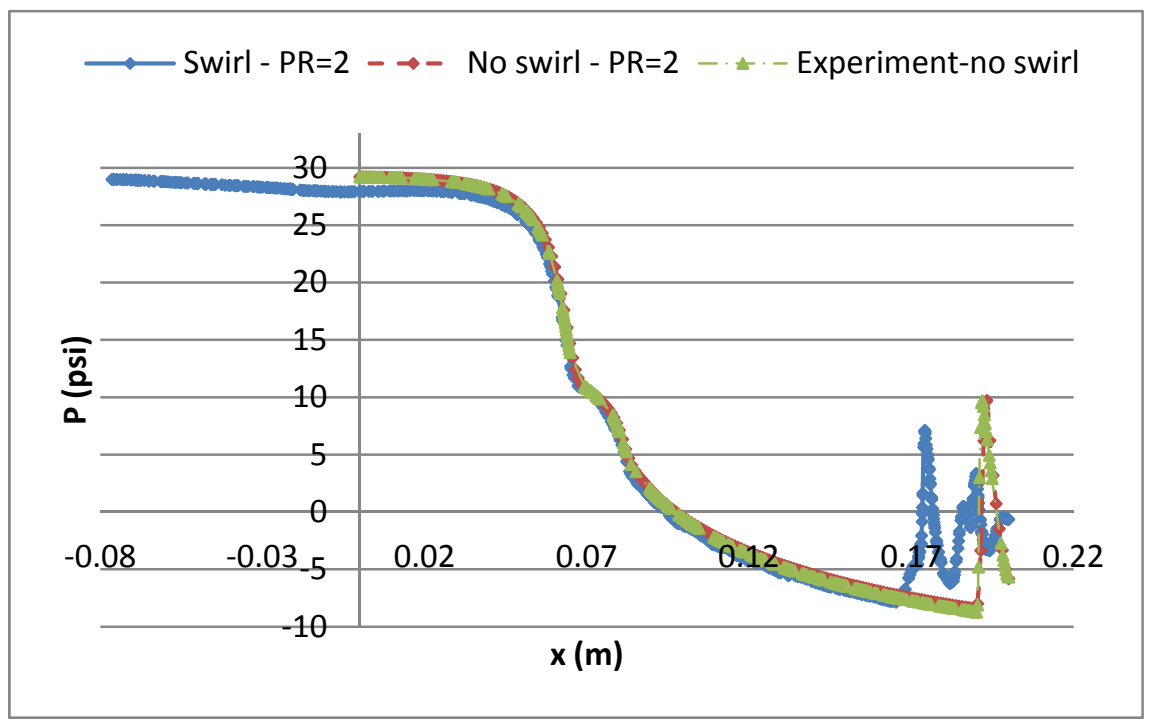

Figure 5: Static pressure diagram for pressure ratio 2 at the centre of nozzle.

For a pressure ratio of 3 the static pressure diagram is shown in Figure 6. We can see that for the nozzle without swirl there is no shock wave inside the nozzle, but by adding swirl conditions, the nozzle remains overexpanded; however, the shock waves become weaker compared to those for a pressure ratio of 2 . The experimental data from Abbasalizadeh [13] for the nozzle without swirl also show that there is no shock wave inside the nozzle and the nozzle is in an underexpanded condition.

Apart from the exit of the nozzle for both pressure ratios the rest of the centre line static pressure of the nozzle with swirl matches that of the nozzle without swirl. 


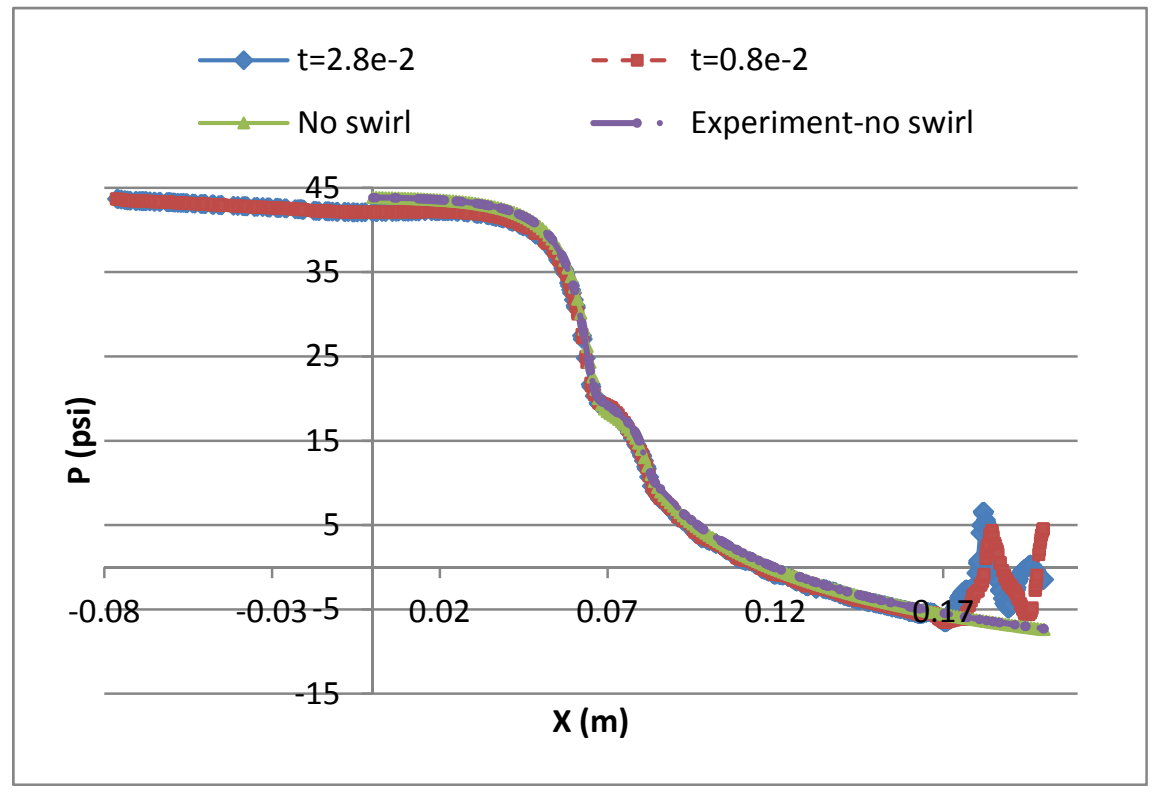

Figure 6: Static pressure diagram for the pressure ratio of 3 at the centre of the nozzle.

\subsection{Numerical validation}

Since it is very difficult to carry out experimental tests inside a nozzle and there are little experimental data for supersonic swirl flow inside a nozzle, the validation process is very important to gain confidence in the results. To check grid dependency all the simulations have been run for $200 \mathrm{k}, 450 \mathrm{k}$ and $800 \mathrm{k}$ cells with refinement at the boundaries and exit of the nozzle to capture the boundary layer and shock waves. We obtain similar results for three different meshes. Considering the computational effort, the results shown here are based on a 400k mesh. The residuals are all reduced at least by an order of 4 . Because of the 3D complex geometry, it was difficult to get a high quality hexahedral mesh; therefore to obtain a good quality mesh a tetrahedral mesh with a prism layer at the boundaries has been used. The minimum quality for the mesh was 0.84 . In addition, the mass flow rate through the inlet and outlet is checked to ensure that conservation of mass is observed. The net mass flow rate through the system is under $1 \%$.

The maximum wall yplus value for the CFD tests was 50, and the RSM which is a core-turbulent model gives better results with a yplus value in the log-low region $(y+>30$ to 60$)$ (Salim and Cheah [14]), so the yplus value is satisfied for most of the nozzle wall. 


\section{Conclusion}

The CFD analysis of swirling flow inside a converging-diverging nozzle is studied at pressure ratios of 2 and 3. For turbulence modelling, the RSM has been used to solve seven Reynolds stress equations. The following conclusions can be drawn:

1. Numerical solutions show swirling flow has unsteady asymmetric behaviour, with a separation zone at the exit of the nozzle, and the main reason for asymmetry is the Coanda effect.

2. Because of anisotropic turbulence, a two equation model will not solve all the scales of turbulent flow, so the RSM with a second order scheme is selected for turbulence modelling.

3. By increasing the pressure ratio to 3 the shock waves move out of the nozzle in no swirl conditions, but by adding swirl condition to the flow the nozzle design criteria will be changed and the nozzle still remains overexpanded.

4. The centre line static pressure shows in swirl conditions that, instead of having one strong shock wave, there are a series of weak shock waves and expansion fans.

\section{References}

[1] Anderson, J. D., Modern compressible flow with historical perspective, New York: McGraw-Hil, 2003.

[2] Anderson, J. D., Hypersonic and high temperature gas dynamics, American Institute of Aeronautics and Astronautics, 2000.

[3] Wilcox, D. C., Turbulence Modelling for CFD, California: Griffin, 1994.

[4] Yajnik, K. and Subbaiah, M., "Experiments on swirling turbulent flows. Part 1. Similarity in swirling flows," Journal of Fluid Mech, vol. 60, no. 4, pp. 665-687, 1973.

[5] Ogor, I. B., Gyllenram, W., Ohlberg, E., Nilsson, H. and Ruprecht, A., "An Adaptive Turbulence Model for Swirling Flow," in Conference on Turbulence and Interactions, Porquerolles, France, 2006.

[6] Shih, T., Liou, W., Shabbir, A., Yang, Z. and Zhu., J., “A New k -e EddyViscosity Model for High Reynolds Number Turbulent Flows - Model Development and Validation," Computers Fluids, vol. 24, no. 3, pp. 227238, 1995.

[7] Xiao, Q., Tsai, H. and Papamoschou, D., "Numerical Investigation of Supersonic Nozzle Flow Seperation," AIAA, pp. 532-541, 2007.

[8] Ansys, “Ansys Fluent 13 User's Guide," Ansys, Inc, 2010.

[9] Daly, B. and Harlow, F. H., "Transport Equations in Turbulence," Physics of Fluids, vol. 13, no. 11, p. 2634-2649, 1970.

[10] Gibson, M. and Launder, B., "Ground Effects on Pressure Fluctuations in the Atmospheric Boundary Layer," Journal of Fluid Mechanics, vol. 86, no. 3, p. 491-511, 1978. 
[11] Sarkar, S. and Balakrishnan, V, "Application of a Reynolds-Stress Turbulence Model to the Compressible Shear Layer," NASA Langley Research Center, Virginia, 1990.

[12] Hadjadj, A. and Onofri, M., "Nozzle Flow Separation," Shock Waves, pp. 163-169, 2009.

[13] Abbasalizadeh, M., Investigation of Three Phase Nozzle flow In an Innovative Sand Blasting System, Chelmsford: Anglia Ruskin University, 2011.

[14] Salim, M. S. and Cheah, S., "Wall yplus Strategy for Dealing with Wallbounded Turbulent Flows," in Proceeding of the International MultiConference of Eng. and Comp., Hong Kong, 2009.

[15] Guo, H.-F., Chen, Z.-Y. and Yu, C.-W., "3D numerical simulation of compressible swirling flow induced by means of tangential inlets," International Journal For Numerical methods in Fluids, pp. 1285-1298, 2008.

[16] Najafi, A., Saidi, M., Sadeghipour, M. and Souhar, M., "Numerical analysis of turbulent swirling decay pipe flow," International Communications in Heat and Mass Transfer, pp. 627-638, 2005. 